\title{
Retraction
}

\section{Author-Initiated Retraction: Shao et al., "LING0-1 Regulates Oligodendrocyte Differentiation through the Cytoplasmic Gelsolin Signaling Pathway"}

At the request of the authors, The Journal of Neuroscience is retracting "LINGO-1 Regulates Oligodendrocyte Differentiation through the Cytoplasmic Gelsolin Signaling Pathway" by Zhaohui Shao, Xinhua Lee, Guanrong Huang, Guoqing Sheng, Christopher E. Henderson, Daniel Louvard, Jiho Sohn, Blake Pepinsky, and Sha Mi, which appeared on pages 3127-3137 of the March 22 , 2017 issue.

It was brought to the attention of the authors that there were concerns related to the accuracy of Figure 5 in the Shao et al. (2017) article. In response, the authors reviewed the underlying data relating to this article carefully. The authors were unable to locate records sufficient to corroborate that 6 animals were treated according to the conditions described in the legend accompanying Figure 5 and in the supporting text of the article. The in vivo data in Figure 5 were considered by the authors and reviewers to be an essential element underlying the conclusions of the paper. In their absence, the authors felt that the starting hypothesis was not fully tested. For these reasons, the authors recommended retraction of the article, and we concurred. We offer our most sincere apologies to the readers.

DOI: 10.1523/JNEUROSCI.2476-18.2018 